\title{
Raman Intensity: An Important Tool in the Study of Nanomaterials and Nanostructures
}

\author{
Ph. Colomban* and A. SlodczyK
}

Laboratoire de Dynamique, Interactions et Réactivité LADIR, UMR 7075

CNRS, Université Pierre et Marie Curie-Paris 6, 2 rue Dunant, 94320 Thiais, France

\begin{abstract}
Analysis of the relative and absolute Raman intensity is very important and powerful tool which allows to understand and characterize the modifications of the crystal/amorphous structure due to: (i) changes of the symmetry, e.g. substitution of the B site ion by the rare earth/lanthanide one and incorporation of mobile species in the case of high temperature protonic conducting perovskites; (ii) changes of the short/long range order, e.g. existence/disappearance of the nanoregions in the case of $\mathrm{PbMg}_{1 / 3} \mathrm{Nb}_{2 / 3} \mathrm{O}_{3-x} \mathrm{PbTiO}_{3}(\mathrm{PMN}-\mathrm{PT})$ relaxor ferroelectric perovskites; (iii) changes of the nanostructure, e.g. depolymerisation of the $\mathrm{Si}-\mathrm{O}$ network due to the substitution of the $\mathrm{Si}^{4+}$ ions (and associated covalent bonds) by the $\mathrm{M}^{+}$cations (forming ionic bonds) or by the incorporation of the metal nanoprecipitates in the case of glasses, glazes and enamels.
\end{abstract}

PACS numbers: 78.30.-j, 77.84.Dy, 61.43.Fs

\section{Introduction}

Raman spectroscopy probing the molecular and crystal lattice vibrations and hence being bottom up approach of the matter appears as one of the most powerful techniques in the study of nanomaterials and nanostructures. The analysis of the Raman mode wave numbers, widths and intensities allows us to obtain the information about the compositions, chemical environment, bonding and crystalline/amorphous structure of a sample material $[1,2]$. Most of the studies consider the evolution of peaks wave numbers and widths, for example as the function of temperature and/or polarization effects. Recently, Raman intensity measurements and band shape analysis are used mainly to determine quantitatively the amount and distribution of different phases in a material, i.e. the Raman mapping [1-3]. In our opinion the study of absolute and relative Raman intensity is also a very powerful tool. The Raman intensity is a function of the polarizability and symmetry and therefore probes the bonding covalence and structure.

The aim of this paper is then to present some recent results which illustrate this approach in order to investigate and understand the changes of the crystal/amorphous structure. Additionally, this paper completes the review recently published considering the relationship between the Raman signature, grain size and mechanical properties of nanosized or nanostructured materials [2]. The Raman intensity analysis allows us characterizing the complex physical behaviour for better possible industrial application in the case of perovskites: high temperature protonic conductors [4] and ferroelectric relax-

\footnotetext{
* corresponding author; e-mail: philippe.colomban@glvt-cnrs.fr
}

ors $\mathrm{PbMg}_{1 / 3} \mathrm{Nb}_{2 / 3} \mathrm{O}_{3-x} \mathrm{PbTiO}_{3}$ (PMN-PT) solid solutions [5]. This analysis is also essential to determine the composition, technology and age of the Cultural Heritage objects made of glass, glaze and enamel or to understand and optimize optical devices made of glass containing dispersed metal nanoparticles [6-12].

\section{Experimental}

The Raman spectra of the perovskite materials were excited with different wavelengths of the $\mathrm{Ar}^{+}$ $\mathrm{Kr}^{+}, \mathrm{He}-\mathrm{Ne}$ and Nd:YAG lasers and recorded in the backscattering geometry using the XY Raman micro-spectrometer (Dilor, France) equipped with the double monochromator filter and a back-illuminated liquid nitrogen cooled CCD detector as well as the high sensitivity LabRAM "Infinity" (Jobin-Yvon-Horiba, France) Raman micro-spectrometer including Notch filters, equipped with a back-illuminated CCD cooled by the Peltier effect.

The Raman spectra of the Cultural Heritage objects were recorded at the laboratory (see above) or on site, for example at the Sainte Chapelle, Paris, using different mobile Raman spectrometers. These spectrometers are equipped with a Peltier effect-cooled CCD, a Jobin Yvon SuperHead with the Edge filter and two few meters long optical fibres, $532 \mathrm{~nm}$ Coherent YAG laser and laptop computer.

Origin Peak Fitting software was used to decompose the Raman spectra following the procedure described in our previous works $[3,5,7]$.

\section{Results and discussion 3.1. Perovskites}

The perovskite structure (general formula $\mathrm{ABO}_{3}$ ) is made of two sublattices: a covalent bonded lattice formed 
of $\mathrm{BO}_{6}$ octahedra sharing their oxygen atoms and an A-cation lattice for which the Coulombian interactions are dominant. The Raman spectroscopy allows following the modifications of these two sublattices, separately. The Raman signature of the perovskite can be described as that made of the covalent entities constituting the structure - $\mathrm{BO}_{6}$ octahedron. The other ions (A-ions) are highly ionic and contribute to the translation oscillation modes which couple themselves with other vibrational modes of the neighbouring entities: translations and rotations/libration of iono-covalent $\mathrm{BO}_{6}$ leading to the wave number shifts. Low wave number region (100-250 $\left.\mathrm{cm}^{-1}\right)$ chiefly involves cationic network and the lattice modes, while a $250-800 \mathrm{~cm}^{-1}$ spectral range reveals the bending and stretching modes of the covalent bonded octahedron $[3,4]$.

\subsubsection{Doping, distortions and electronic defects}

The perovskite materials are very well known due to their vast properties and corresponding application. For example the compounds described by the following formula $\mathrm{A}^{2+}(\mathrm{Ba}, \mathrm{Sr}, \mathrm{Ca}$ etc. $) \mathrm{B}^{4+}(\mathrm{Zr}, \mathrm{Ce}$, Ti etc. $) \mathrm{O}_{3}$ belong to the family of high temperature protonic conductors and might be used as membranes of the solid oxide fuel cell and water electrolyser $[4,13,14]$. The presence of protons is not intrinsic to their structure. Therefore, they must be first doped with a few mol\% of trivalent cations (lanthanides $(\mathrm{Ln})$ or rare earths $(\mathrm{RE})$ ) at the $\mathrm{B}$ site that results in the formation of the oxide ion vacancies. When these oxygen-deficient perovskites are annealed at the requested temperature in the presence of water vapour the oxygen vacancies are filled by the protonic moieties. Substituting the B element, formation of the oxygen vacancies and presence of the protons modify significantly the host perovskite structure.

Figure 1 presents the Raman spectra of pure, Ln-modified and protonated Ln-modified $\mathrm{BaZrO}_{3}$. These Raman spectra consist of number of broad, not intense bands [4]. The presence of the Raman spectrum for these materials is contradictory with a description of the X-ray diffraction pattern with the cubic symmetry and the Pm3m space group ([4] and references therein), because for such cubic symmetry the first-order Raman activity is forbidden $[15,16]$. This suggests that the symmetry, at least at the local Raman scale within the analyzed spot $\left(\approx\right.$ few $\left.\mu \mathrm{m}^{2}\right)$, is lower than the cubic mean one. The observed Raman spectrum is assigned to have its source in the nanodomains with the symmetry different than cubic one. Similar results were presented by Lucazeau [16] and Chemarin et al. [17] for $\mathrm{BaZrO}_{3}$. The authors showed that these nanodomains are very sensitive to the changes of high pressure. Namely, as pressure increases (up to $9 \mathrm{GPa}$ ) the nanodomains are forced to interact more strongly which leads to a continuous structure with long range order. The absolute Raman intensity decreases continuously and finally at $9 \mathrm{GPa}$ the Raman spectrum disappears, which is in a very good agreement with the "ideal" cubic symmetry determined by the $\mathrm{X}$-ray diffraction.
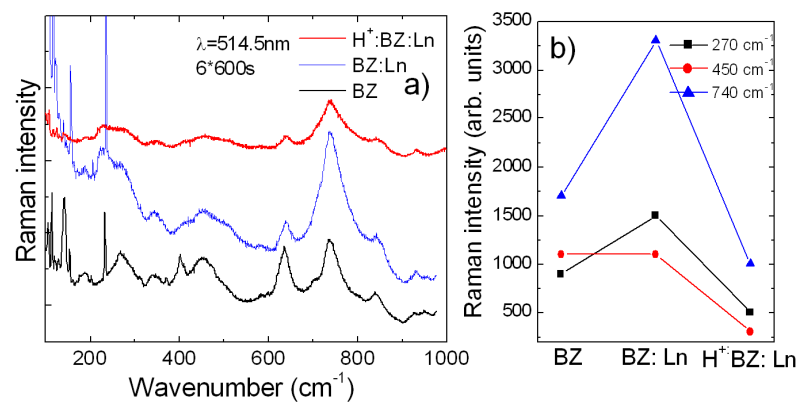

Fig. 1. (a) Raman spectra of pure, Ln-modified and protonated Ln-modified barium zirconates. (b) Changes of the absolute Raman intensity recorded for $\mathrm{BaZrO}_{3}$ after Ln-substitution and then after protonation (after [18]).
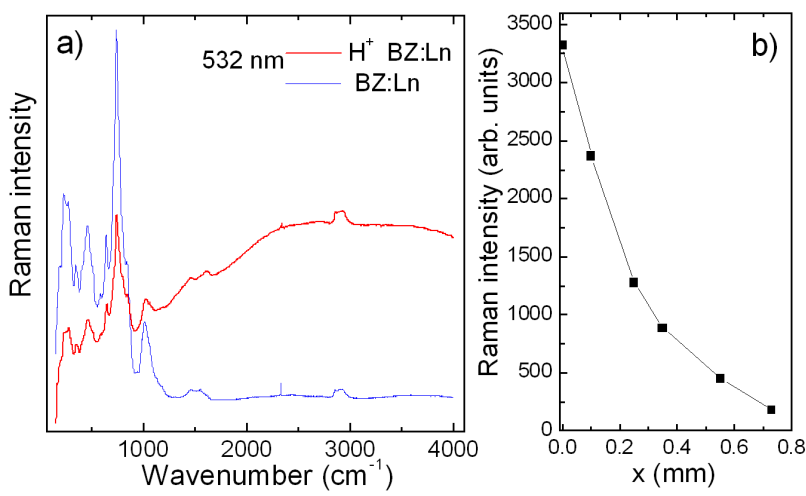

Fig. 2. (a) The comparison between the Raman spectra recorded for protonated and non-protonated $\mathrm{BaZrO}_{3}: \mathrm{Ln}$. (b) The changes of absolute Raman intensity recorded for a protonated $\mathrm{BaZrO}_{3}: \mathrm{Ln}$ pellet in different spots from the ceramic surface till the centre/heart (distance from 0 till $0.78 \mathrm{~mm}$ ). See [18] for details.

As can be seen in Fig. 1, the significant differences of the absolute intensity values are detected for pure, Ln/RE-modified and protonated materials. Namely, the increase in intensity is observed after the partial substitution of $\mathrm{Zr}$ by $\mathrm{Ln} / \mathrm{RE}$. Since the intensity allows one evaluating the distortions of the crystal structure, it shows that the doping and associated oxygen vacancies enhance the distortions of the host lattice. The X-ray diffraction data showing the changes of the unit cell volume between the pure, doped and protonated samples confirm the presence of such distortions [18]. After the protonation, a strong intensity decrease is observed. This suggests that the crystal structure is "re-symmetrised" due to the presence of the protonic species. Additionally, after the proton incorporation a broad component centred at $2500 \mathrm{~cm}^{-1}$ appears (Fig. 2a). Despite the nature of this broad band is ambiguous: fluorescence related to the dangling bonds, the so called A, B, C bands [14] or new phenomena connected with the formation of elec- 
tronic defects associated to ionic proton (polaron?) or combination of all of these phenomena, it is very useful from experimental point of view [18]. Namely, its intensity is correlated to the protonic species content and their special space distribution. Figure $2 \mathrm{~b}$ presents the band intensity recorded in different spots on the section fracture of a protonated pellet from surface of ceramic pellet till the centre. As can be seen the distribution of the protonic species in the ceramic is not constant: surface is very well protonated while in the centre there is almost no protonic species as expected from Fick's diffusion laws.

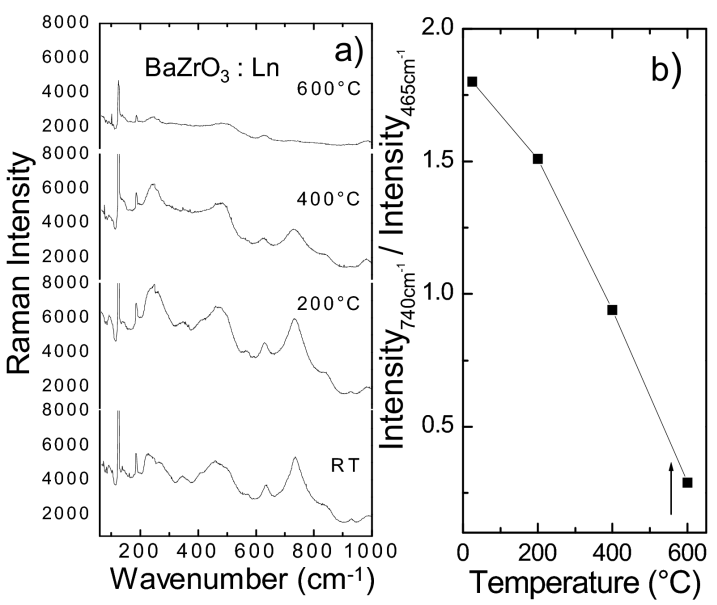

Fig. 3. (a) Temperature evolution of the Ln-modified $\mathrm{BaZrO}_{3}$ Raman spectra. (b) The presence of the structural phase transition is manifested by the relative intensity ratio of the stretching mode $\approx 740 \mathrm{~cm}^{-1}$ and the bending one $\approx 465 \mathrm{~cm}^{-1}$. See [4] for details.

Figure 3 shows the temperature evolution of the Raman spectra of $\mathrm{BaZrO}_{3}: \mathrm{Ln}$. The Raman data point to the presence of the structural phase transition between 400 and $600^{\circ} \mathrm{C}$ in this compound [4]. This phase transition can be better manifested by the relative intensity ratio of the stretching mode located near $740 \mathrm{~cm}^{-1}$ vs. the bending one near $465 \mathrm{~cm}^{-1}$ (see Fig. 2). These results show clearly the efficiency of the relative Raman intensity to analyse pre- and post-phase transition behaviour.

\subsubsection{Nanoregions in the PMN-PT crystals and ceramics}

Another very interesting example of the perovskites are the $\mathrm{PbMg}_{1 / 3} \mathrm{Nb}_{2 / 3} \mathrm{O}_{3-x} \mathrm{PbTiO}_{3}(\mathrm{PMN}-\mathrm{PT})$ solid solutions. Since they possess the outstanding dielectric and electromechanical properties i.e. giant piezoeffect [19], they provide an excellent opportunity for improvements in the sonar application. These superior properties are characteristic for the solid solutions from the so-called morphotropic phase boundary, i.e. the region of the phase diagram with the 30-38\% PT content, and result from the complex, not well understood, physical behaviour [5]. The structural studies reveal the coexistence of different symmetries ordered for different scale, the nonpolar matrix with the chaotically embedded polar nanoregions.
Figure 4a presents the Raman spectra of room temperature (RT) poled PMN-34.5\% PT textured ceramic recorded in a wide temperature range. It is noteworthy that our studies confirm that the properties of the textured ceramics are very similar to those ones characteristic for single crystals. This result is very important from the application point of view and allows proposing the textured ceramic as the low cost alternative of the single crystal [5]. The Raman spectra presented in Fig. 4a are typical of relaxors. Namely, they consist of a number of very broad bands observed in the whole measured temperature range, even within the paraelectric cubic phase with the Pm3m space group. The presence of these Raman modes within the cubic symmetry, similar as for $\mathrm{BaZrO}_{3}$, reveals the existence of the nanoregions with the symmetry lower than this cubic one.
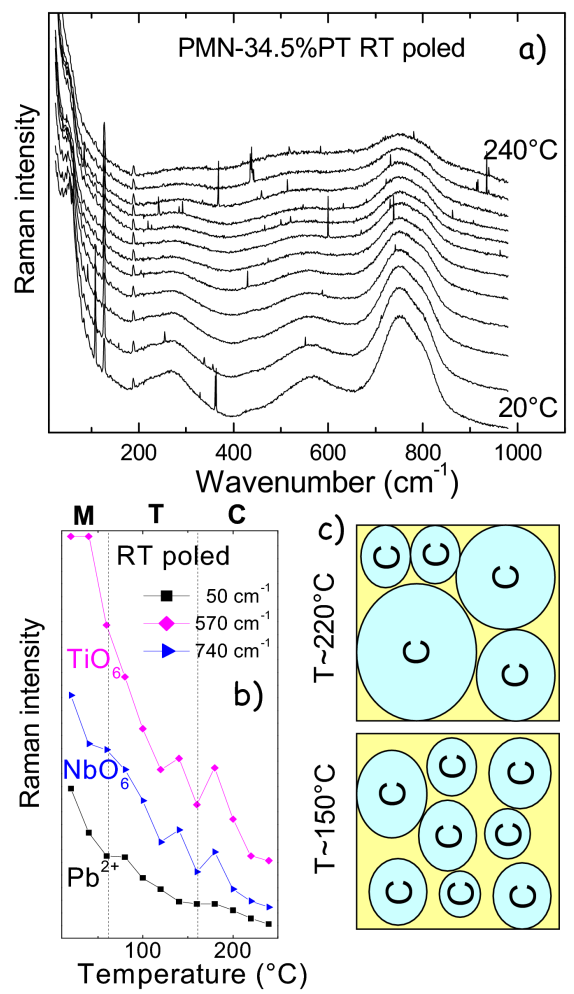

Fig. 4. (a) Raman spectra of RT poled PMN-34.5\%PT textured ceramic recorded in a wide temperature range (temperature step $=20^{\circ} \mathrm{C}$ ) (after [5]). (b) Temperature evolution of the absolute Raman mode intensities. Dashed lines correspond to the structural phase transition sequence: monoclinic $(\mathrm{M})$ - tetragonal $(\mathrm{T})-\mathrm{cu}-$ bic (C). (c) Sketch of the continuous symmetry evolution towards the cubic one. More interpenetrated topology is likely.

As can be seen in Fig. 4 the Raman spectra do not change much with the temperature increase except the intensity values. Let us note that the all Raman spectra were recorded in the same conditions. Figure $4 \mathrm{~b}$ shows the temperature dependences of the Raman mode intensities. The absolute intensity of all modes decreases 
significantly with the temperature increase. In general, the integrated intensity is proportional to the temperature [16], this relation was very well satisfied for example in the case of natural diamond up to $1800 \mathrm{~K}[20]$. Therefore, the shut down of intensity with the temperature increasing can be explained by the changes of the short/long range order correlations as the function of the nanoregion organization. As graphically sketched in Fig. 4c when the temperature increases, the local symmetry evolves continuously towards the cubic one. This result is similar to that obtained for $\mathrm{BaZrO}_{3}$ as the function of high pressure $[16,17]$. Additional, this fits well with the phase transition determined in the thermal expansion measurements, close to $200^{\circ} \mathrm{C}$ [5], and thus shows that the true cubic symmetry can be achieved only at rather high temperature, i.e. much above the maximum of dielectric permittivity.

\subsection{Glassy silicates}

\subsection{1. $\mathrm{SiO}_{4}$ network and polymerization index}

Pure silica can be described as a 3D network of the $\mathrm{SiO}_{4}$ tetrahedra sharing their oxygen atoms and forming the $\mathrm{Si}-\mathrm{O}-\mathrm{Si}$ bridges. Its density is then maximal and a melting requires very high temperature. All applications in the science, art and technology of glasses, glazes and enamels consist of a controlled modification of the 3D $\mathrm{Si}-\mathrm{O}$ network by replacement of $\mathrm{Si}^{4+}$ strong covalently bonded atoms by ionically bonded $\mathrm{M}^{+} / \mathrm{M}^{2+}(\mathrm{Ca}, \mathrm{Na}, \mathrm{K}$, $\mathrm{Pb})$. This decreases the number of $\mathrm{Si}-\mathrm{O}$ bridges and the connectivity of network, i.e. the so-called depolymerisation of the $3 \mathrm{D} \mathrm{Si-O}$ network takes place. In consequence, the glass nanostructure as well as all characteristic glass properties are modified, for example the melting temperature decreases [6-10].

Cultural Heritage objects carry information in their micro/nanostructure regarding their provenance, production process as well as even their conservation conditions. Leading experts generally deduced all these information basing on their personal sensory perceptions. Since it is possible to obtain the same visual effects like colour, transparency, etc. using totally different technological process the greater objectivity is mandatory for correct identification, dating, etc. Our studies show that the Raman spectroscopy offers a very potential tool to characterize the different Cultural Heritage objects made of glass [6-12, 21-24].

The Raman spectrum of a silicate consists as a first approximation solely of the signature of the covalent $\mathrm{Si}-\mathrm{O}$ network, the $\mathrm{Al}-\mathrm{O}$ bond contribution is too poor to be detected because of its ionic character. In a typical Raman spectrum of silicate two parts can be distinguished: the so-called boson peak and the molecular signature of the different $\mathrm{SiO}_{4}$ entity. The boson peak roughly corresponds to the projection of lattice/external modes of the $\mathrm{SiO}_{4}$ and it is highly coupled to the translations of the ionically bonded elements. The molecular signature consists of two multiplets, the $\mathrm{SiO}_{4}$ bending modes centred at $\approx 500 \mathrm{~cm}^{-1}$ and the $\mathrm{SiO}_{4}$ stretching ones located at $\approx 1000 \mathrm{~cm}^{-1}$.
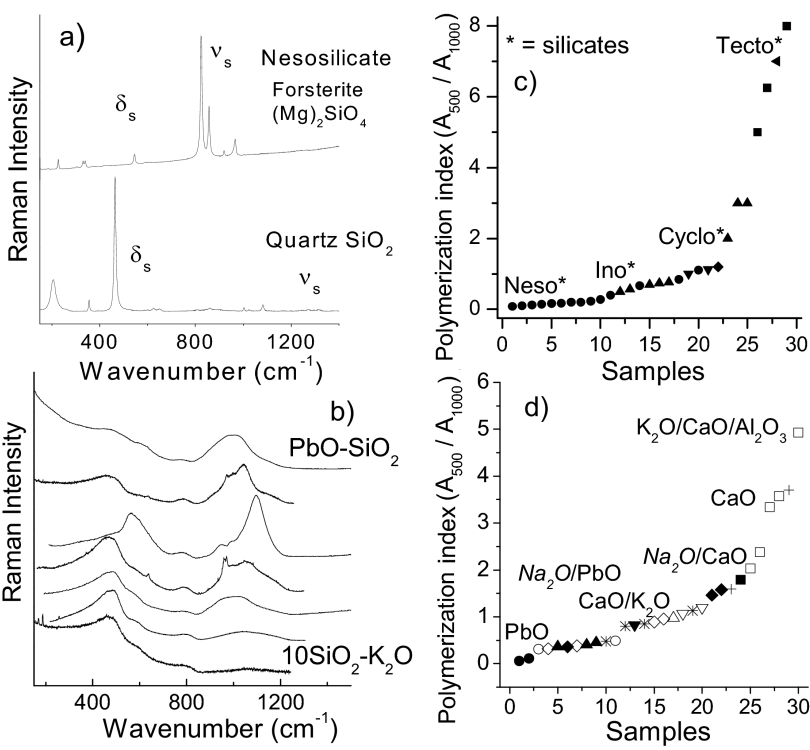

Fig. 5. Raman spectra of representative crystalline (a) and amorphous (b) silicates with significant intensity differences between the $\mathrm{SiO}_{4}$ bending and stretching modes. Plot of the polymerization index: (c) for series of crystalline silicates, the low values are observed for nesosilicates while the high for silica-rich-tectosilicates, (d) for series of glasses and glazes with the increasing silica content and different nature of flux; note that for a given type of compound the flux content decreases from left to right. See [6] for details.

The substitution of $\mathrm{Si}$ atoms by $\mathrm{Na}, \mathrm{K}$, $\mathrm{Ca}$ and $\mathrm{Pb}$ ones changes not only the $\mathrm{Si}-\mathrm{O}$ connectivity but also the partial ionic charge of terminal oxygen atoms which modifies the Raman cross-section of bending $\left(\approx 500 \mathrm{~cm}^{-1}\right)$ and stretching $\left(\approx 1000 \mathrm{~cm}^{-1}\right)$ massifs. Therefore, exact positions, intensity and shape of the molecular multiplets reveal the different tetrahedra arrangements of the modified $\mathrm{Si}-\mathrm{O}$ network. Based on the so-called $\mathrm{Q}_{n}$ model the Raman scattering offers a differentiation guide of all silicates compositions [9]. In the Raman spectrum characteristic of $\mathrm{Pb}$-rich glaze the $\approx 1000 \mathrm{~cm}^{-1}$ massif dominates significantly whereas for silica rich material a very strong band at $500 \mathrm{~cm}^{-1}$ is observed (Fig. 5b). Such intensity variations are recorded as well for the crystalline silicates (Fig. 5a). It is well established that silicates can be classified as a function of their tetrahedral network type as nesosilicates (isolated $\mathrm{SiO}_{4}$ tetrahedra, $\mathrm{Q}_{0}$ ), sorosilicates $\left(\mathrm{Q}_{1}\right)$, cyclosilicates, innosilicates $\left(\mathrm{Q}_{2}, \mathrm{Q}_{3}\right)$, and tectosilicates $\left(\mathrm{Q}_{4}\right)$ and this type of classification can be extended to the local conformation of $\mathrm{SiO}_{4}$ tetrahedra of the glass nanostructure $[8,9]$. For crystalline and amorphous silicates, the ratio of the area of the $\mathrm{Si}-\mathrm{O}$ bending modes $\left(\mathrm{A}_{500}\right)$ vs. the area of the $\mathrm{Si}-\mathrm{O}$ stretching ones $\left(\mathrm{A}_{1000}\right)$ measures the polymerization degree $[8,9]$, the so-called polymerization index $I_{\mathrm{p}}$ is defined. The value of the polymerization index $\left(I_{\mathrm{p}}\right)$ reveals the glass nanostructure and compositions: $I_{\mathrm{p}}<0.8$ - lead-rich 
glasses and nesosilicates, $I_{\mathrm{p}} \approx 1.1-\mathrm{Na}$ glasses and inosilicates, $I_{\mathrm{p}} \approx 1.3-2.5-\mathrm{Ca}$ glasses and cyclosilicates, $I_{\mathrm{p}}>2.5-$ K-rich glasses and tectosilicates, $I_{\mathrm{p}} \approx 5-6-$ porcelain glazes and $I_{\mathrm{p}} \approx 7$ - Quartz and amorphous silica (see Fig. 5c and d) [7]. Variations of the $\mathrm{Si}-\mathrm{O}-\mathrm{Si}$ angle with polymerization and neighbouring cations also shift the wave number of the bending multiplets and are correlated to the intensity of the different $\mathrm{Q}_{n}$ components of the $1000 \mathrm{~cm}^{-1}$ multiplet.

The calculation of the relative intensity, i.e. determination of polymerization index, can be employed as the identification method, namely the characteristic glass compositions reveal the features of the technology process used during a certain period for example Roman, Medieval, 19th century restoration, etc. [21-24].

\subsubsection{Corrosion and dating}

The analysis of the absolute Raman intensity offers also very powerful tool to date the different objects kept in rather similar conditions. For a given compositions and the same recording conditions the absolute intensity of a Raman spectrum is the function of the optical properties of the analyzed materials, mainly the colour and clearness [9]. If material is not transparent for a laser wavelength the absorption takes place. The intensity of the scattered light can decrease significantly because of the weathering effects such as the appearance of microcracks, pits, salts, precipitates and corroded silica rich layer at the upper surface exposed to different pollutions (Fig. 6a). It is noteworthy that the glass weathering is
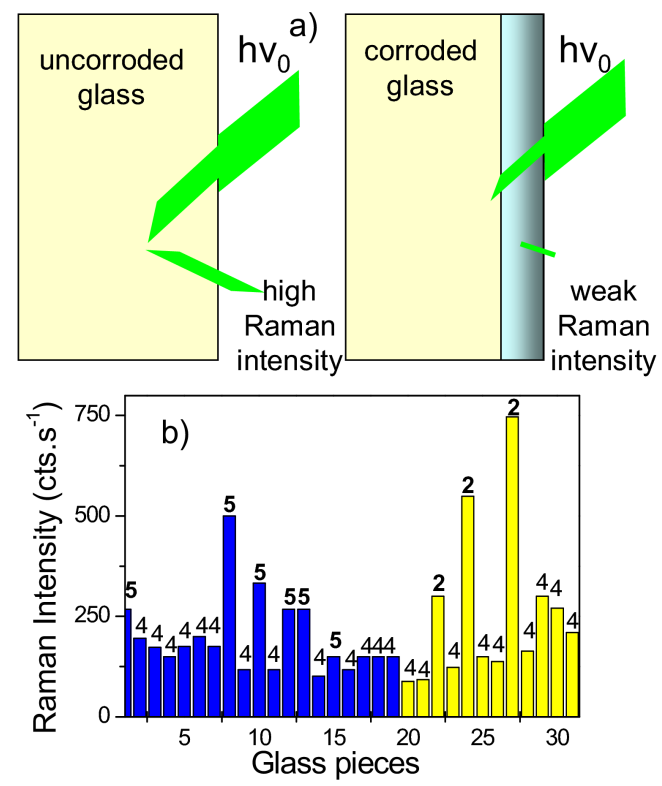

Fig. 6. (a) Sketch explaining the changes of absolute Raman intensity observed for uncorroded and corroded glasses. (b) Differences of the absolute Raman intensity recorded for Sainte Chapelle stained glasses: 2 - uncorroded or 5 - low-corroded Na-based glasses (19th restoration), 4 - corroded K-based glasses (Middle Age). See [9] for details. more important for K-rich glasses [25, 26]. Figure 6b shows the absolute intensity measurements of the Sainte Chapelle stained glasses. As it can be easily seen, the old, lixiviated glasses (corroded K-based glasses) [9] characteristic for Middle Age exhibit not intense Raman signatures whereas the pieces dating to the 19th century restoration (low- to uncorroded Na-based glasses) preserve rather well their optical qualities and show much higher intensity values.

\subsubsection{Plasmon coupling in metal nanoparticle- -containing glasses}

Another important changes of the absolute intensity values are observed in the glasses with the dispersed metal nanoparticles such as $\mathrm{Cu}^{0}$ or $\mathrm{Ag}^{0}$ [8]. The metal nanoparticles are used as a common "dye" (lustre glazed pottery, red flashed and yellow stained glasses), photonic crystal, optical guide and filters [27-29]. The particular colour depends on the redox state of metal, for example, the glasses with dispersed $\mathrm{Cu}^{2+}$ have a blue colour, $\mathrm{Cu}^{0}$ are red, whereas $\mathrm{Cu}^{+}$are colourless. Figure 7 presents the Raman spectra of the red coated glass $\left(\mathrm{Cu}^{0}\right)$, a 19th century copy of the red middle age glass, recorded using two different wavelengths. For such dispersed metal nanoparticles a very specific UV-visible absorption curve is obtained (surface plasmon resonance (SPR) peak) as shown in Fig. 7a and the Raman intensity is drasti-

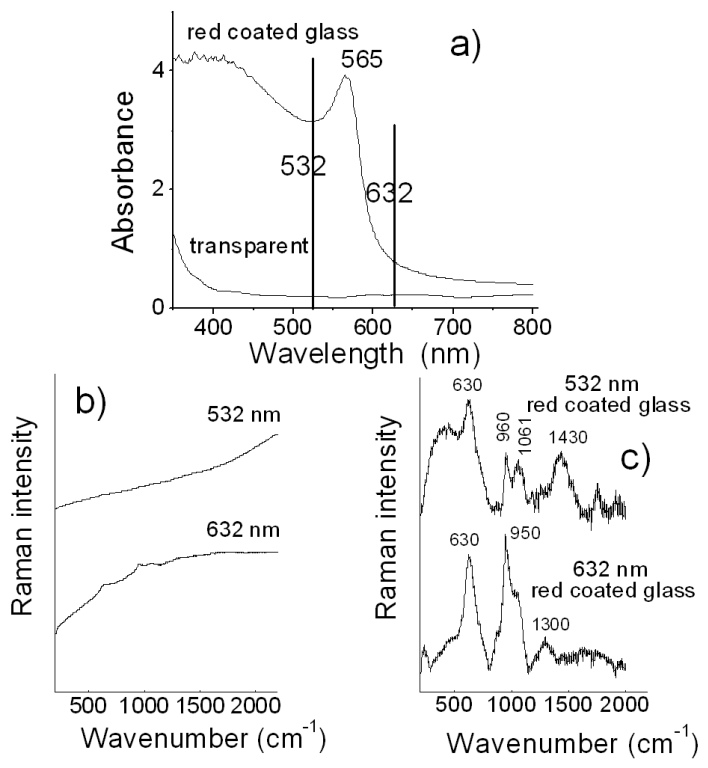

Fig. 7. (a) The comparison of the visible absorption spectra of transparent and red coated glasses; green $(532 \mathrm{~nm})$ and red $(632 \mathrm{~nm})$ laser excitation lines are marked as well as the plasmon peak at $565 \mathrm{~nm}$. (b) Raman spectra of the red coated glasses (red colour due to the dispersed $\mathrm{Cu}^{0}$ nanoparticles) recorded using the two above wavelengths. (c) The same Raman spectra but after the base line subtraction. Let us note the changes of relative intensity of bending and stretching massifs. See [8] for details. 
cally decreased $[7,8,12]$ for wavelength below the energy of the SPR peak. Comparison of the spectra recorded with $(532 \mathrm{~nm})$ and without $(632 \mathrm{~nm})$ the resonant condition evidences the difference between the mean signature of the whole glassy network (632 $\mathrm{nm}$ excitation) and the local signature of the network surrounding the $\mathrm{Cu}^{0}$ nanoparticle (532 $\mathrm{nm}$ excitation). Changes are due to the depolymerization of the $\mathrm{Si}-\mathrm{O}$ network around the metal nanoparticle. Better spectra are obtained with the model materials when the $\mathrm{Cu}^{0}$ content is very small in order to limit the light absorption [8].

\section{Conclusion}

The limited examples discussed above show clearly that the analysis of relative and absolute Raman intensity is a very powerful tool to go deeper in the understanding of the different materials and their properties. This requires controlled procedure and the use of the same instruments to reach a good reliability. The development of standard references covering a large excitation wavelengths domain is requested to develop this approach.

\section{References}

[1] Ph. Colomban, Spectroscopy Europe 15, 8 (2003).

[2] G. Gouadec, Ph. Colomban, Prog. Cryst. Growth Charact. Mater. 53, 1 (2007).

[3] M. Havel, D. Baron, Ph. Colomban, J. Mater. Sci. 39, 6183 (2004).

[4] A. Slodczyk, Ph. Colomban, D. Lamago, M.H. Limage, F. Romain, S. Willemin, B. Sala, Ionics 14, 215 (2008).

[5] A. Slodczyk, Ph. Colomban, M. Pham-Thi, J. Phys. Chem. Solids 69, 2503 (2008).

[6] Ph. Colomban, J. Non-Cryst. Solids 323, 180 (2003).

[7] Ph. Colomban, A. Tournié, L. Bellot-Gurlet, J. Raman Spectrosc. 37, 841 (2006).

[8] Ph. Colomban, H.D. Schreiber, J. Raman Spectrosc. 36, 884 (2005).

[9] Ph. Colomban, A. Tournié, J. Cultural Heritage 8, 242 (2007).

[10] Ph. Colomban, in: Raman Spectroscopy in Archaeology and Art History, Eds. H.G.M. Edwards, J.M. Chalmers, Royal Society of Chemistry, London 2005, Ch. 13, p. 192.
[11] Ph. Colomban, F. Romain, A. Neiman, I. Animitsa, Solid State Ionics 145, 339 (2001).

[12] Ph. Colomban, J. Nano Research, in press.

[13] H. Iwahara, in: Proton Conductors, Ed. Ph. Colomban, Cambridge University Press, Cambridge 1992, Ch. 34, p. 511.

[14] Ph. Colomban, Proton Conductors, 2nd ed., Cambridge University Press, Cambridge 2008.

[15] A.E. Pasto, R.A. Condrate, The Laser Raman Spectra of Several Perovskite Zirconates, Adv. Spectrosc., Vol. 1, Ed. J.P. Mathieu, Heyden, London 1973.

[16] G. Lucazeau, J. Raman Spectrosc. 34, 478 (2003).

[17] C. Chemarin, N. Rosman, T. Pagnier, G. Lucazeau, J. Solid State Chem. 149, 298 (2000).

[18] A. Slodczyk, Ph. Colomban, S. Willemin, O. Laxroix, B. Sala, submitted to J. Raman Spectrosc. 40,513 (2009).

[19] S.E. Park, T.R. Shrout, J. Appl. Phys. 82, 1804 (1997).

[20] H. Herchen, M.A. Capelli, Phys. Rev. B 43, 11740 (1991).

[21] N.Q. Liem, N. Thanh, Ph. Colomban, J. Raman Spectrosc. 33, 287 (2002).

[22] Ph. Colomban, V. Milande, J. Raman Spectrosc. 37, 606 (2006).

[23] Ph. Colomban, J. Cultural Heritage (2008), doi: 10.1016/j.culher.2008.06.005.

[24] Ph. Colomban, in: MRS Fall Meeting Proc., Vol. 852, Eds. P. Vandiver, J. Mass, A. Murray, 2004, p. 153.

[25] Ph. Colomban, M.P. Etcheveny, M. Asquier, M. Bounichou, A. Tournie, J. Raman Spectrosc. 37, 614 (2006).

[26] A. Tournie, P. Ricciardi, Ph. Colomban, Solid State Ionics, doi: 10.1016/j.ssi.2008.07.019.

[27] Ph. Colomban, V. Vendange, in: MRS Fall Meeting Proc., Vol. 457, Eds. S. Komarneni, J.C. Parher, H.J. Wollenberger, 1997, p. 451.

[28] U. Kreisig, M. Vollmer, Optical Properties of Metal Cluster, Springer, Berlin 1995.

[29] M. Wohler, Adv. Semi-Cond. Mag. 18, 46 (2005). 